\title{
A formação em Enfermagem para a prática da gestão: revisão integrativa
}

\author{
Training in Nursing for management practice: integrative review \\ Formación en Enfermeira para la práctica de gestión: revisión integrativa
}

Recebido: 17/12/2021 | Revisado: 23/12/2021 | Aceito: 29/12/2021 | Publicado: 30/12/2021

\author{
Ana Vitória Andrade de Sousa Mendes \\ ORCID: https://orcid.org/0000-0002-4066-8991 \\ Centro Universitário Christus, Brasil \\ E-mail: enfanavitoriaandrade@gmail.com \\ Mariana Keli Ferreira dos Santos Hansen \\ ORCID: https://orcid.org/0000-0002-9679-7389 \\ Centro Universitário Christus, Brasil \\ E-mail: mkfs.mariana@gmail.com \\ Alisson Salatiek Ferreira de Freitas \\ ORCID: https://orcid.org/0000-0002-4547-5785 \\ Centro Universitário Christus, Brasil \\ E-mail: salatiek@gmail.com \\ Eugênio Santana Franco \\ ORCID: https://orcid.org/0000-0001-5962-6743 \\ $\mathrm{PhD}$ em Desenvolvimento de Tecnologia em Saúde, Brasil \\ E-mail: eugeniofgf@hotmail.com \\ Maria Tayslane Crispim de Souza \\ ORCID: https://orcid.org/0000-0002-4687-3136 \\ Centro Universitário Christus, Brasil \\ E-mail: maria.tayslane.crispim@gmail.com \\ Clara Emillyn Alves de Araújo \\ ORCID: https://orcid.org/0000-0002-5123-1453 \\ Centro Universitário Christus, Brasil \\ E-mail: claraemillyn@gmail.com \\ Roberta Balreira Arrais Maia \\ ORCID: https://orcid.org/0000-0002-9679-7389 \\ Centro Universitário Christus, Brasil \\ E-mail: udvroberta@gmail.com \\ Eveline Silva Lima \\ ORCID: https://orcid.org/0000-0003-0568-6374 \\ Centro Universitário Christus, Brasil \\ E-mail: evelinesils@gmail.com \\ Deborah Pedrosa Moreira \\ ORCID: https://orcid.org/0000-0003-4313-2479 \\ Centro Universitário Christus, Brasil \\ E-mail: deborah.moreira@unichristus.edu.br
}

\begin{abstract}
Resumo
Objetivo: Identificar nas evidências científicas os temas abordados sobre a prática da gestão na formação em enfermagem. Metodologia: Revisão integrativa da literatura, com coleta de dados no mês de setembro de 2021, nas seguintes bases de dados: Pubmed, LILACS (Literatura Latino-Americana e do Caribe em Ciências da Saúde) e BDEnf. Resultados: Do total de 6 artigos, todos foram realizados no Brasil, a partir de análises textuais discorremos sobre temáticas como: formação e necessidades do SUS, educação permanente e a necessidade de formar enfermeiros para a área, disciplinas curriculares, metodologia, teoria e prática e enfermagem para a gestão. Conclusão: O estudo verificou as dificuldades na aproximação/vínculo entre as instituições de ensino superior e os serviços de saúde, possibilitou a descoberta do diário de campo como uma ferramenta positiva no processo de aprendizagem e confirmou-se que as atividades práticas assim como nas demais temáticas são vistos como essenciais para a experiência da gestão.
\end{abstract}

Palavras-chave: Gestão em saúde; Enfermagem; Educação; Educação superior.

\begin{abstract}
Objective: Identify in the scientific evidence the themes addressed about the practice of management in nursing education. Methodology: Integrative literature review, with data collection in September 2021, in the following databases: Pubmed, LILACS (Latin American and Caribbean Literature on Health Sciences) and BDEnf. Results: Of a total of 6 articles, all were carried out in Brazil, from textual analysis, we discuss themes such as: formation and needs of the SUS, permanent education and the need to train nurses for the area, curriculum subjects, methodology, theory
\end{abstract}


and practice and nursing for management. Conclusions: The study verified the difficulties in the approximation/link between higher education institutions and health services, enabled the discovery of the field diary as a positive tool in the learning process and it was confirmed that practical activities, as well as in other themes, are seen as essential for the management experience.

Keywords: Health management; Nursing; Education; Higher education.

\section{Resumen}

Objetivo: Identificar en la evidencia científica los temas abordados sobre la práctica de la gestión en la educación en enfermería. Metodología: Revisión integrativa de la literatura, con recolección de datos en septiembre de 2021, en las siguientes bases de datos: Pubmed, LILACS (Literatura Latinoamericana y del Caribe en Ciencias de la Salud) y BDEnf. Resultados: De un total de 6 artículos, todos fueron realizados en Brasil, a partir del análisis textual, se discuten temas como: formación y necesidades del SUS, la formación permanente y la necesidad de formar enfermeras para el área, asignaturas curriculares, metodología, teoría y práctica, y enfermería para la gestión. Conclusiones: El estudio verificó las dificultades en la aproximación / vinculación entre las instituciones de educación superior y los servicios de salud, posibilitó el descubrimiento del diario de campo como una herramienta positiva en el proceso de aprendizaje y se confirmó que las actividades prácticas, así como en otros temas, se consideran fundamentales para la experiencia de gestión.

Palabras clave: Gestión de la salud; Enfermería; Educación; Educación superior.

\section{Introdução}

A formação em áreas da Saúde e na Enfermagem tem-se demonstrado com uma crescente tendência e busca. E apesar das políticas implementadas, com as Diretrizes Curriculares Nacionais e das estratégias de Gestão da Educação na Saúde estabelecidas até a atualidade, a formação em Enfermagem ainda apresenta desafios não só históricos, como também contemporâneos (Ximenes, et al., 2020).

Nesse sentido, o Art. $4^{\circ}$ da Resolução CNE/CES nº 3, de 7 de novembro de 2001, que institui as Diretrizes Curriculares Nacionais do Curso de Graduação em Enfermagem, no que diz respeito a formação do enfermeiro, tem por objetivo dotar o profissional de conhecimentos requeridos para o exercício de competências e habilidades gerais, como a gestão (Brasil, 2001). O Exame Nacional de Desempenho dos Estudantes (ENADE) nas suas seis edições incentivou o aluno à tomada de decisões através das resoluções dos casos e problemas de saúde dentro do cenário da saúde pública, ou dentro da área hospitalar (Messias, et al., 2021) favorecendo a interface com um dos pilares da gestão.

Nesse contexto, pesquisadores elegem o gerenciamento como uma prática dinâmica e complexa. Necessitando de uma comunicação efetiva, para estabelecer e fortalecer vínculos entre: a equipe, os agentes do cuidado e os usuários dos serviços. Destacando-se também, a importância de habilidades de análise crítica para a tomada de decisão (Barreto, et al., 2018).

Sendo assim, durante a graduação do curso de enfermagem, o formando necessita vivenciar uma boa base de conhecimento solidificada e firme, abordando metodologias significativas, transformadoras e que o qualifiquem, visando melhorias à sociedade no qual o mesmo está inserido (Ximenes, et al., 2020).

Dito isto, dentre as competências e habilidades gerais como base de conhecimentos necessários à gestão, destaca-se a Liderança no trabalho em equipe multiprofissional, no qual os profissionais de saúde deverão estar aptos a assumir posições de liderança, tendo em vista o bem-estar da comunidade envolvendo o compromisso, a responsabilidade, a empatia, habilidade para tomada de decisões, a comunicação e o gerenciamento de forma efetiva e eficaz (Brasil, 2001).

As estratégias didáticas utilizadas nos cursos de Enfermagem que enfatizem a articulação entre teoria e prática, priorizam a ênfase ao planejamento estratégico situacional. O que pode significar que o ensino de gestão em enfermagem está acompanhando as mudanças pedagógicas da educação superior na área da saúde (Santos et al., 2018), colaborando para o reforço para a aptidão a se tornarem empreendedores, gestores, empregadores ou líderes na equipe de saúde.

Contudo, a competência gerencial deve-se basear também nas experiências individuais do ambiente em que se atua, e levando em conta as necessidades deste. Ressalta-se que o aprimoramento de habilidades deve ocorrer ainda na graduação, e o 
curso de Enfermagem ainda é um dos poucos que possuem na área da saúde, diretrizes curriculares com disciplinas voltadas para a administração (Tenório, et al., 2019).

Diante disso, o presente estudo tem o objetivo de identificar nas evidências científicas as estratégias sobre a formação em enfermagem para a prática da gestão.

\section{Método}

Uma revisão integrativa da literatura trata-se da construção de uma análise ampla dos estudos já publicados, contribuindo nas discussões sobre os métodos e os resultados das pesquisas, além de refletir sobre a realização de prováveis futuros estudos. No geral, é preciso desenvolver seis etapas distintas para a construção de uma revisão integrativa (Mendes, Silveira \& Galvão, 2008). As quais descreveremos a seguir:

\section{Primeira etapa: Identificação do tema e seleção da hipótese ou questão norteadora da pesquisa.}

A pergunta para esta pesquisa foi realizada através da estratégia PICO, que representa um acrônimo para Paciente, Intervenção, Controle ou comparação e "Outcomes" (desfecho/resultados em inglês), acreditando-se que uma pergunta de pesquisa bem desenvolvida e adequada, contribui para uma definição correta de evidências, orientando a construção da pergunta de partida, norteando a busca bibliográfica e contribuindo na localização de forma mais precisa e rápida (Santos et al., 2007). Sendo assim, obtivemos a seguinte questão norteadora: Como as evidências científicas abordam a prática da gestão na formação em enfermagem? E sua definição aplicada pela estratégia PICO na tabela a seguir.

Quadro 1: Definição da estratégia PICO.

\begin{tabular}{|l|l|l|}
\hline Acrônimo & Definição & Descrição \\
\hline P & Paciente ou problema & Discente de enfermagem \\
\hline I & Intervenção & Qualidade da formação em enfermagem \\
\hline C & Controle ou comparação & Intervenções na formação para a prática da gestão \\
\hline O & Desfecho (“outcomes") & Competências para a prática da gestão \\
\hline
\end{tabular}

Fonte: Dados da pesquisa (2021).

\section{Segunda etapa: Estabelecimento de critérios para inclusão e exclusão de estudos/ amostragem ou busca na literatura.}

No mês de setembro de 2021 foi realizada uma busca para o levantamento das evidências científicas nas seguintes bases de dados: Pubmed, LILACS (Literatura Latino-Americana e do Caribe em Ciências da Saúde) e BDEnf., em companhia da orientadora, coorientadora e da coautora do presente estudo, através de uma reunião virtual em uma plataforma digital.

Para busca dos artigos foram utilizados os seguintes descritores: Health Management AND Students, Nursing AND Education, Nursing AND Education, Higher, na língua inglesa, por ter maior amplitude nos resultados. Sendo respectivamente Gestão em Saúde E Estudantes, Enfermagem E Educação, Enfermagem E Educação Superior, sua tradução na língua portuguesa, e Gestión en Salud Y Estudiantes, Enfermería Y Educación, Enfermería Y Educación Superior, a tradução na língua espanhola. Este processo ocorreu de forma desafiadora, visto que, inicialmente a partir dos descritores poucos estudos eram selecionados, até que a partir destas palavras chaves em questão conseguimos um número maior, e as definimos.

Foram adotados os seguintes critérios de inclusão: artigos originais, nacionais e internacionais, com textos disponíveis na íntegra, nos idiomas português, inglês ou espanhol, sem delimitação de intervalo de tempo, que de alguma forma contribuísse com o tema direcionado a formação em enfermagem para a prática da gestão. Foram excluídos estudos como: teses de mestrado, revisão de literatura, trabalhos publicados em congressos e anais, editoriais e artigos repetidos. 


\section{Terceira etapa: definição das informações a serem extraídas dos estudos selecionados/ categorização dos estudos.}

As informações a serem extraídas dos estudos selecionados, utilizam, em geral de algum determinado instrumento que reúne e sintetiza as informações (Mendes, et al., 2008). Sendo assim, como técnica para coleta de dados após a definição dos artigos incluídos na pesquisa, utilizamos o Instrumento de Avaliação do Rigor Metodológico das Pesquisas Selecionadas, adaptado de Critical Appraisal Skills Programme (CASP)- Programa de habilidades em leitura crítica. @ Milton Keynes Primary Care Trust 2002 (Healthcare, 2013).

\section{Quarta etapa: avaliação dos estudos incluídos na revisão integrativa}

Nesta etapa utilizamos o Método de HEIS (Hierarchy Of Evidence For Intervention Studies) que é a classificação hierárquica das evidências para avaliação dos estudos, em sete níveis, no qual iremos incluir os que estiverem nos níveis IV, V, e VI, que correspondem respectivamente a evidências provenientes de estudos de coorte e de caso-controle bem delineados, evidências originárias de revisão sistemática de estudos descritivos e qualitativos, e, evidências derivadas de um único estudo descritivo ou qualitativo (Sousa et al., 2010).

\section{Quinta etapa: interpretação dos resultados}

Nesta é realizada a discussão dos principais resultados encontrados na pesquisa, onde ocorre a comparação com a teoria, identificação de conclusões e implicações da revisão integrativa. Através da revisão é possível perceber possíveis fatores que afetam a política e a prática da enfermagem, e ao identificar essas lacunas nos permite o apontamento de sugestões importantes para futuras pesquisas visando a melhoria da assistência da enfermagem (Mendes, et al., 2008).

\section{Sexta etapa: apresentação da revisão/síntese do conhecimento}

Os estudos analisados foram apresentados em forma de quadro com a síntese dos conteúdos encontrados em cada artigo incluso, produzindo um impacto importante devido ao acúmulo dos conhecimentos existente sobre a temática em questão (Mendes, et al., 2008).

\section{Resultados}

A seguir apresentam-se os resultados obtidos das buscas, sendo demonstrado o processo na Figura 1, que após busca inicial apenas com os descritores já citados, obtivemos 100 publicações pela Pubmed, e 46 na Lilacs/BVS/BDEnf. Após a seleção dos artigos pelo critério de avaliação de títulos, este número caiu para 5 publicações pela Pubmed, e 13 pela Lilacs/BVS/BDEnf., Em seguida através da leitura dos títulos, resumos e artigos lidos na íntegra, selecionamos os que de alguma forma contribuíssem a responder à pergunta norteadora da revisão, no qual chegamos ao número final do total de 6 artigos incluídos. A Figura 1 demonstra o fluxograma da coleta e análise dos dados. 
Figura 1 - Gráfico de Hierarquia Rotulada com o fluxograma da coleta e análise dos dados.

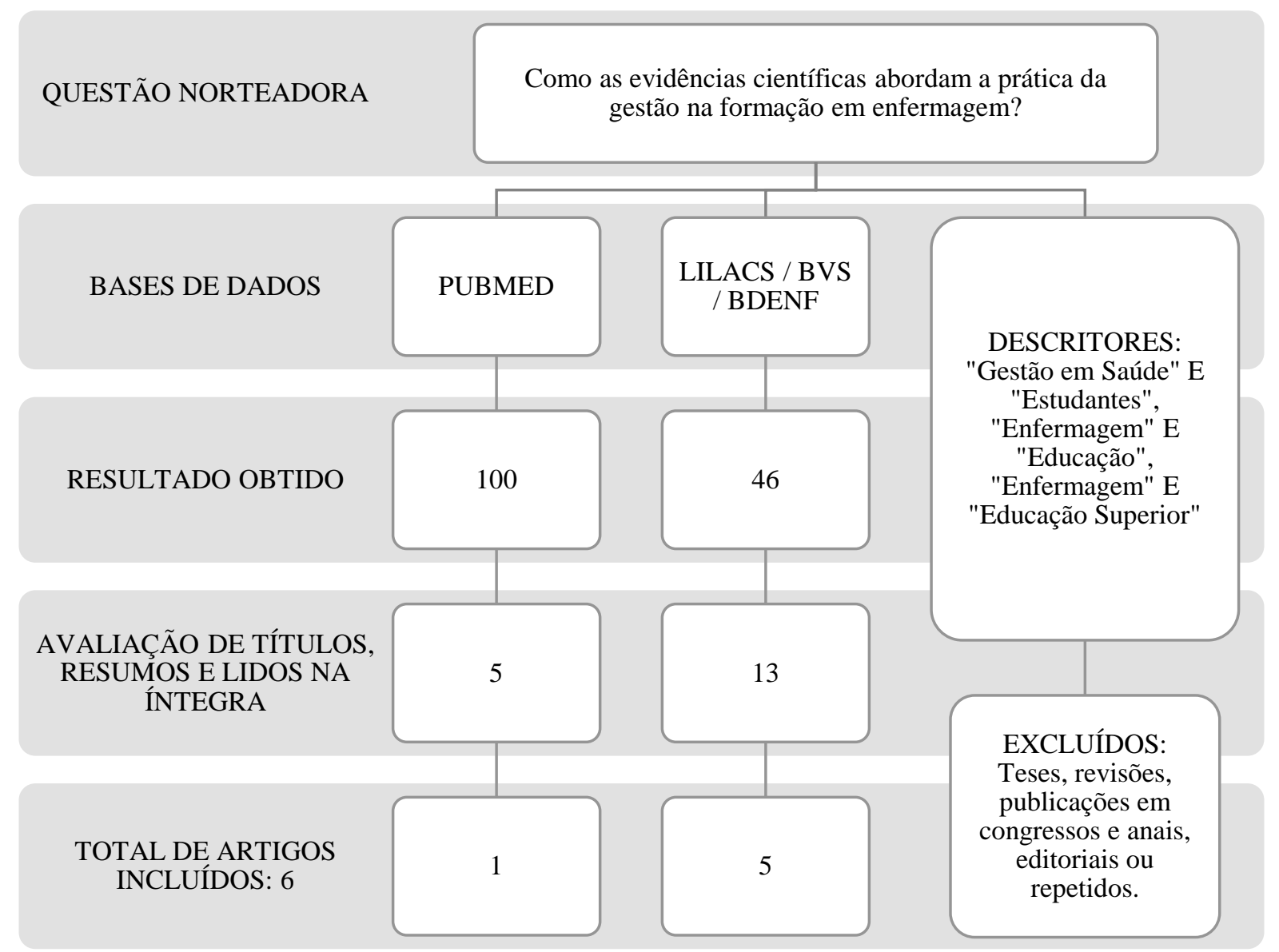

Fonte: Dados da pesquisa (2021).

Todos os 6 artigos foram realizados no Brasil sendo: um no Rio de Janeiro, dois em São Paulo, dois em Goiânia e um em Salvador. No estudo todos obtiveram o resultado em Nível A do Critical Appraisal Skills Programme (CASP), um Instrumento de Avaliação do Rigor Metodológico das Pesquisas Selecionadas, com pontuação que os classificam com uma boa qualidade metodológica e viés reduzido. O Quadro 2 apresenta dados de cada estudo e as variáveis analisadas. 
Quadro 2 - Distribuição dos artigos por títulos, autores, periódico, base de dados, ano, local de publicação, nível de evidência e principais achados.

\begin{tabular}{|c|c|c|c|c|}
\hline $\mathbf{N}^{\circ}$ & Título & Autores & $\begin{array}{l}\text { Periódico / Base / Ano } \\
\text { / Local / Nível de } \\
\text { Evidência }\end{array}$ & Principais Achados \\
\hline A1 & $\begin{array}{l}\text { A formação de enfermeiros } \\
\text { e sua aproximação com os } \\
\text { pressupostos das Diretrizes } \\
\text { Curriculares Nacionais } \\
\text { (DCN) e da Atenção Básica }\end{array}$ & $\begin{array}{ll}\text { Magnago } & \mathrm{C}, \\
\text { Pierantoni CR. } & \end{array}$ & $\begin{array}{l}\text { Ciência e Saúde } \\
\text { Coletiva / LILACS e } \\
\text { BDENF / 2020 / Rio de } \\
\text { Janeiro-RJ / IV }\end{array}$ & $\begin{array}{l}\text { Formação distante das necessidades, estrutura curricular } \\
\text { centrada em disciplinas fragmentadas nos ciclos básicos e } \\
\text { profissionalizante relacionado ao recomendado pelas DCNs. } \\
\text { Perfil de competências potente para o desenvolvimento de } \\
\text { ações de promoção, prevenção, gerenciamento e técnicas de } \\
\text { enfermagem, estando previstas no escopo de competências e } \\
\text { habilidades gerais que segundo as DCNs são compatíveis } \\
\text { com as principais demandas assistenciais da atenção básica } \\
\text { (Magnago \& Pierantoni, 2020). }\end{array}$ \\
\hline A2 & $\begin{array}{l}\text { Ações assistenciais e } \\
\text { gerenciais desenvolvidas } \\
\text { no Estágio Curricular } \\
\text { Supervisionado: impressão } \\
\text { dos atores envolvidos }\end{array}$ & 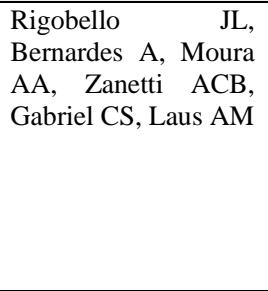 & $\begin{array}{l}\text { Revista da Escola de } \\
\text { Enfermagem / } \\
\text { PUBMED / } 2018 \text { / São } \\
\text { Paulo-SP / IV }\end{array}$ & $\begin{array}{l}\text { O desenvolvimento das ações assistenciais e de } \\
\text { gerenciamento foi positivo, porém uma parcela dos egressos } \\
\text { apontou que o Estágio Curricular Supervisionado não } \\
\text { proporcionava ao aluno desenvolver todas as atividades } \\
\text { realizadas pela equipe de enfermagem. Também que a } \\
\text { assistência integral à saúde, a segurança técnica profissional, } \\
\text { precisavam de interferência na dinâmica de trabalho, } \\
\text { necessitando de adequações estruturais (Rigobello, et al., } \\
\text { 2018). }\end{array}$ \\
\hline $\mathbf{A 3}$ & $\begin{array}{l}\text { O diário de campo utilizado } \\
\text { como estratégia de ensino e } \\
\text { instrumento de análise do } \\
\text { trabalho da enfermagem }\end{array}$ & $\begin{array}{l}\text { Soares AN, Silveira } \\
\text { APO, Silveira BV, } \\
\text { Vieira JS, Souza } \\
\text { LCBA, Alexandre LR } \\
\text { et al. }\end{array}$ & $\begin{array}{l}\text { Revista Eletrônica de } \\
\text { Enfermagem / LILACS } \\
\text { e BDENF / 2011/ } \\
\text { Goiânia-GO / VI }\end{array}$ & $\begin{array}{l}\text { Diante das consequências do método funcional, o estilo de } \\
\text { liderança da coordenadora de enfermagem e questões éticas } \\
\text { relacionadas à assistência. Considera-se que o diário } \\
\text { permitiu aos alunos articularem teoria e prática, } \\
\text { evidenciando dificuldades enfrentadas no cotidiano dos } \\
\text { serviços de saúde e confronto com o conhecimento } \\
\text { produzido (Soares, et al., 2011). }\end{array}$ \\
\hline A4 & $\begin{array}{l}\text { Observação e diário de } \\
\text { campo: técnicas utilizadas } \\
\text { no estágio da } \\
\text { administração } \\
\text { enfermagem }\end{array}$ & $\begin{array}{lr}\text { Freitas } & \text { MEA, } \\
\text { Spagnol } & \text { CA, } \\
\text { Camargos AT } & \end{array}$ & $\begin{array}{l}\text { Revista Baiana de } \\
\text { Enfermagem / LILACS } \\
\text { e BDENF / 2006 / } \\
\text { Salvador-BA / VI }\end{array}$ & $\begin{array}{l}\text { Emergiram quatro categorias, das quais evidenciavam que a } \\
\text { estratégia utilizada possibilitou aos alunos uma reflexão } \\
\text { sobre o exercício profissional da enfermagem articulando a } \\
\text { teoria e a prática, oportunizou sua inserção nos serviços de } \\
\text { saúde e facilitou a introdução em seu futuro ambiente de } \\
\text { trabalho (Freitas, Spagnol \& Camargos, 2006). }\end{array}$ \\
\hline A5 & $\begin{array}{lr}\text { Perfil dos egressos } & \text { de } \\
\text { gerenciamento } & \text { de } \\
\text { enfermagem } & \text { dos } \\
\text { Programas da área de } \\
\text { Enfermagem da Região Sul }\end{array}$ & $\begin{array}{l}\text { Erdmann AL, } \\
\text { Andrade SR, Santos } \\
\text { JLG, Oliveira RJT }\end{array}$ & $\begin{array}{l}\text { Revista da Escola de } \\
\text { Enfermagem / LILACS } \\
\text { e BDENF / } 2011 \text { / São } \\
\text { Paulo-SP / V }\end{array}$ & $\begin{array}{l}\text { Os programas de graduação em Enfermagem da Região Sul } \\
\text { titularam } 409 \text { alunos, sendo } 129(31,5 \%) \text { nas linhas de } \\
\text { pesquisa de gestão/gerenciamento em enfermagem, } 116 \\
(89,9 \%) \text { mestres e } 13(10,1 \%) \text { doutores que atuam } \\
\text { majoritariamente na docência. Dos mestres titulados, dois } \\
(1,7 \%) \text { já são doutores e } 39(33,6 \%) \text { estão cursando o } \\
\text { doutorado (Erdmann, et al., } 2011) \text {. }\end{array}$ \\
\hline A6 & $\begin{array}{l}\text { Uso de metodologia ativa } \\
\text { na disciplina } \\
\text { gerenciamento } \\
\text { enfermagem em saúde } \\
\text { coletiva da FEO/UFPEL }\end{array}$ & $\begin{array}{l}\text { Heck RM, Jardim } \\
\text { VR, Dilélio AS, Silva } \\
\text { SJ }\end{array}$ & $\begin{array}{l}\text { Revista Eletrônica de } \\
\text { Enfermagem / LILACS } \\
\text { e BDENF / 2009 / } \\
\text { Goiânia-GO / VI }\end{array}$ & $\begin{array}{l}\text { Os acadêmicos destacam a aproximação com o mundo do } \\
\text { trabalho na lógica do SUS, com ferramentas como o } \\
\text { planejamento, organização, coordenação, compreensão da } \\
\text { hierarquização e regionalização dos serviços de saúde no } \\
\text { município e região. O acadêmico é motivado a exercitar a } \\
\text { crítica e reflexão em relação aos indicadores de saúde, à } \\
\text { gestão de recursos humanos, físicos e financeiros e tomar } \\
\text { decisões indicando o que é prioridade para cada um contexto } \\
\text { específico (Heck, et al., 2009). }\end{array}$ \\
\hline
\end{tabular}

Fonte: Dados da pesquisa (2021).

O quadro a seguir apresenta uma síntese geral dos principais resultados encontrados no presente estudo, classificados por temas e subtemas de acordo com a que pertencem cada um. 
Quadro 3 - Síntese dos resultados de acordo com os estudos, temas e subtemas a que pertencem.

\begin{tabular}{|c|c|c|}
\hline Tema & Subtema & Resultados Principais \\
\hline \multirow{3}{*}{$\begin{array}{l}\text { Formação } \\
\text { Enfermagem }\end{array}$} & $\begin{array}{l}\text { Formação e } \\
\text { necessidades } \\
\text { do SUS }\end{array}$ & $\begin{array}{l}\text { - Estabelecer uma articulação entre as instituições formadoras e o SUS tem sido um desafio persistente } \\
\text { (Magnago \& Pierantoni, 2020). } \\
\text { - A maior ênfase do processo formativo do enfermeiro é atenção básica (Magnago \& Pierantoni, 2020). } \\
\text { - Planejamento da carreira e investimento na qualificação profissional em enfermagem (Erdmann, et al., } \\
\text { 2011). } \\
\text { - Programas de pós-graduação contribuem com o avanço e consolidação do conhecimento científico, } \\
\text { tecnológico e de inovação na gestão/gerência de enfermagem (Erdmann, et al., 2011). } \\
\text { - Projetar um profissional atuante na gestão/gerência em saúde e enfermagem, com uma formação } \\
\text { competente, visionária, empreendedora e construtora de políticas públicas, os programas de pós-graduação dão } \\
\text { um passo avante sobre as mudanças esperadas pela sociedade (Erdmann, et al., 2011). }\end{array}$ \\
\hline & $\begin{array}{l}\text { Percepção } \\
\text { das } \\
\text { Disciplinas }\end{array}$ & $\begin{array}{l}\text { - O estágio curricular supervisionado oportuniza ao aluno se autodescobrir, fortalecer compromissos e } \\
\text { responsabilidades, aprender e executar competências de liderança e trabalho em equipe (Rigobello, et al., 2018). } \\
\text { - O estágio auxilia na construção da aprendizagem das dimensões assistencial e gerencial bem como a } \\
\text { relação entre elas (Rigobello, et al., 2018). } \\
\text { - No estágio os estudantes desenvolvem competências gerenciais e capacidades para diagnosticar problemas } \\
\text { de saúde e estratégias de intervenção respeitando princípios éticos e legais (Rigobello, et al., 2018). } \\
\text { - A partir da experiência os alunos compreendem a disposição de leitos, exames laboratoriais, custo do } \\
\text { cuidado diário, importância do seguimento dos protocolos, fatura de procedimentos, financiamento etc. (Heck, } \\
\text { et al., 2009). }\end{array}$ \\
\hline & $\begin{array}{l}\text { Metodologia } \\
\text { aplicada à } \\
\text { Enfermagem }\end{array}$ & $\begin{array}{l}\text { - Percepção dos alunos sobre os exemplos durante a aula expositiva e atribuindo peso maior a vivência } \\
\text { proporcionada pelos dias de estágio na observação participativa (Freitas, et al., 2006). } \\
\text { - Alunos apontaram que o diário de campo é uma prática que possibilita exercitar a escrita de forma mais } \\
\text { sistematizada de registros e anotações dos diversos instrumentos utilizados pela enfermagem (Freitas, et al., } \\
\text { 2006). } \\
\text { - Debates do que se apresenta como problema prioritário mais urgente a ser superado, exercitando o } \\
\text { planejamento estratégico situacional (Heck, et al., 2009). }\end{array}$ \\
\hline \multirow[t]{2}{*}{ Prática da Gestão } & $\begin{array}{l}\text { Percepção } \\
\text { sobre Teoria } \\
\text { e Prática }\end{array}$ & $\begin{array}{l}\text { - Oportunidade durante o estágio e escrita do diário de campo de analisar o estilo de liderança da } \\
\text { coordenação de enfermagem do ambulatório (Soares, et al., 2011). } \\
\text { - Necessidade de mudar valores e buscar modelos gerenciais que enfatizem atitudes humanizadas e ações } \\
\text { de qualidade (Soares, et al., 2011). } \\
\text { - Percepção sobre a metodologia do trabalho da enfermagem, riscos ocupacionais, comunicação } \\
\text { interpessoal, ética e a lei do exercício profissional (Freitas, et al., 2006). } \\
\text { - Deparar-se durante o estágio com conflitos pessoais que oportunizam amadurecimento pessoal e } \\
\text { profissional (Freitas, et al., 2006). }\end{array}$ \\
\hline & $\begin{array}{l}\text { Enfermagem } \\
\text { para a Gestão }\end{array}$ & $\begin{array}{l}\text { - As competências administrativas e gerenciais são preconizadas pelas diretrizes curriculares de } \\
\text { enfermagem e são características do trabalho do enfermeiro, que se mostra mais preparado para assumir tais } \\
\text { funções (Magnago \& Pierantoni, 2020). }\end{array}$ \\
\hline
\end{tabular}

Fonte: Dados da pesquisa (2021).

\section{Discussão}

A partir da análise detalhada dos estudos, ao contrário do que se esperava, verificou-se um baixo volume de publicações sobre o assunto abordado utilizando esses descritores, seja para o bom funcionamento de equipes, setores ou até mesmo instituições nas quais enfermeiros possuam cargos de gerência. Diante disso, obtivemos quatro categorias dos conteúdos dos artigos selecionados.

\section{Formação, educação permanente e necessidades do SUS}

Estudos apontam que durante o preparo profissional o treinamento desenvolvido pelas instituições tem favorecido de forma inferior ao que se espera para o sistema de saúde, e que isto se deve provavelmente ao desafio de estabelecer uma articulação entre o próprio SUS (Sistema Único de Saúde) - com suas necessidades de recursos humanos e as instituições formadoras (Erdmann, et al., 2011, Magnago \& Pierantoni, 2020).

A partir disso, há empecilhos que comprometem a introdução dos educandos na realidade do SUS, como a resistência das unidades em recepcionar os alunos, despreparo profissional, baixa quantidade de docentes e consequentemente preceptores, infraestrutura precária com poucos recursos, além da disputa por diferentes campos de estágio pelas instituições formadoras (Heck, et al., 2009, Magnago \& Pierantoni, 2020). 
Essas dificuldades de relacionamento entre as instituições de ensino superior e dos serviços de saúde para a prática, como por exemplo, no estágio curricular supervisionado acabam limitando o aluno no desenvolvimento de suas atividades assistenciais e gerenciais, consequentemente limitando seu aprendizado (Rigobello, et al., 2018).

É importante também trazermos uma reflexão sobre quais os motivos que levaram essas instituições a tomarem uma postura tão resistente á inclusão dos educandos em sua rotina de trabalho, seja apreensão pelo novo, mudanças nas rotinas, possibilidade de descobertas de protocolos desenvolvidos erroneamente pela instituição ou pelos próprios alunos, postura inadequada dos acadêmicos e/ou preceptores, dentre outras inúmeras possibilidades.

Sabemos que as competências gerenciais e administrativas são preconizadas pelas diretrizes curriculares de enfermagem, sendo características importantes para o trabalho do enfermeiro. A formação destacada e diferenciada, empreendedora e construtora de políticas públicas, torna-se um diferencial deixando o enfermeiro mais preparado para assumir cargos gerenciais (Magnago \& Pierantoni, 2020). O vínculo estreito entre a comunidade científica e serviço gera resultados que beneficiam a todos, pois os acadêmicos aprendem na prática, o serviço oportuniza o recebimento de suporte teórico atualizado e, sobretudo, o usuário, que recebe boa assistência (Silva, et al., 2021).

É imprescindível investir na qualificação dos profissionais, professores e preceptores que acompanham os alunos, nas redes de atenção, e no firmamento de metas e objetivos entre as instituições de ensino e os serviços do SUS (Magnago \& Pierantoni, 2020), principalmente no que se refere a contribuir com o novo ambiente no qual estarão inseridos, seja com ações, implementações de melhorias, protocolos que simplifiquem os processos e tragam maior qualidade no funcionamento para que a partir disto, vínculos possam ser fortalecidos de ambas as partes.

A enfermagem atualmente deve produzir e compartilhar ações direcionadas aos usuários e aos serviços do SUS, com competências, habilidades e conhecimentos atualizados, não apenas nas áreas assistenciais, como também nas gerenciais. O que se torna um desafio, visto a importância dessa área na dimensão do trabalho do enfermeiro, e ampliando suas necessidades de aprimoramento de conhecimentos para além da sua formação em enfermagem, atuando nos cursos de graduação e pós-graduação e investimento em qualificação profissional, somando com a ciência ao produzir conhecimentos relevantes pela inovação em enfermagem e para a sociedade (Soares, et al., 2011, Erdmann, et al., 2011).

Alguns estudos apontaram que durante o processo formativo o tema de gerenciamento na Graduação em Enfermagem é inferiorizado, ou seja, atribuído menor importância em relação as demais categorias (Rigobello, et al., 2018). E isto é um fator que acaba prejudicando diretamente o desenvolvimento e aprendizado de futuros enfermeiros que provavelmente ainda não possuirão uma base sólida para posterior aprimoramento profissional na área.

Diante disso, em uma das pesquisas incluídas, os graduandos relataram a dificuldade de compreender objetivamente o SUS durante a maior parte do curso, até a oportunidade de uma visualização mais concreta do todo, e alguns destes também referiram que a disciplina deveria ser ofertada ainda nos primeiros semestres, alegando que assim, se permitiria melhor compreensão sobre o SUS (Heck, et al., 2009).

\section{Práticas e estágio supervisionado para identificação e desenvolvimento de competências gerenciais}

Um ponto relevante que esteve presente nos artigos, e destacado como de suma importância para o aprendizado do enfermeiro em formação, foi sobre as atividades práticas, que diante da literatura a prática é vista como essencial para a experiência da gestão, assim como também a observação de enfermeiros gestores (Freitas, et al., 2006, Soares, et al., 2011).

O estágio permite o aluno a oportunidade do autodescobrimento, promove a convivência, fortalece compromissos e responsabilidades para se colocar em prática a liderança a comunicação e o trabalho em equipe. Auxilia na construção da aprendizagem das dimensões assistencial e gerencial bem como a relação entre elas (Rigobello, et al., 2018, Magnago \& Pierantoni, 2020). 
Os estudos também trouxeram a percepção dos alunos diante da metodologia do trabalho da enfermagem, reconheceram a estrutura organizacional das instituições de estágio, quanto aos riscos ocupacionais, comunicação interpessoal, ética e a lei do exercício profissional etc. Além de permitir a interdependência com os demais campos do conhecimento da saúde, educação e gestão, com a produção e a atuação multi, inter e transdisciplinar (Freitas, et al., 2006, Erdmann, et al., 2011).

Historicamente, apesar dos esforços, a associação entre teoria e prática se encontra desvinculada, acarretando pouca aplicabilidade prática sobre a teoria. A manutenção deste fenômeno sobre a estrutura curricular se apresenta de forma insatisfatória diante das diretrizes curriculares de enfermagem. O ensino e o trabalho são necessários para que a partir de sua aproximação, competências se desenvolvam de forma que contribuam com o profissional na busca por melhorias da qualidade de vida da população (Magnago \& Pierantoni, 2020).

Alguns estudos trazem, como achados advindo dos próprios educandos a atribuição de maior impacto positivo no aprendizado do que apenas nas exposições de casos clínicos durante a aula expositiva, e apontaram a necessidade de buscar modelos e valores gerenciais que executem ações de qualidade através de atitudes humanizadas (Freitas, et al., 2006, Soares, et al., 2011).

Esses valores podem ser desenvolvidos por meio de competências técnico-científicas através de metodologias ativas que colabora para a construção de um sujeito ativo, crítico e reflexivo, com maior autonomia no seu conhecimento (Garcia, et al., 2018) e o estágio curricular supervisionado possibilita o desenvolvimento do raciocínio crítico, de habilidades de comunicação, liderança e tomada de decisões no mundo real do trabalho (Esteves, et al., 2018). Somado a isso, as metodologias ativas e participativas, que favorecem a participação e criticidade, são as principais escolhas.

Quando o aluno passa pela experiência prática nos campos de estágio, sejam estes curriculares ou extracurriculares, eles se deparam com as demandas diferenciadas, rotinas, e um extenso conjunto de fatores que o fazem amadurecer, pessoal e profissionalmente. $\mathrm{O}$ contato com os gestores, profissionais dos mais diversos setores, com os pacientes e acompanhantes, trazem uma nova perspectiva para o indivíduo, seja na forma de se comunicar, de abordar as pessoas, descobrir o momento certo de ouvir e o de falar, de intervir ou aguardar, todas essas características e muitas outras são desenvolvidas e aprimoradas também a partir destas vivências.

O Diário de Campo foi considerado pelos estudos como uma ferramenta que permite a articulação entre teoria e prática ao expor dificuldades nas rotinas dos serviços e os enfrentar com o conhecimento produzido previamente, ou seja, articulação a partir da visão crítica das cenas observadas no cotidiano com as concepções teóricas estudadas (Freitas, et al., 2006, Soares, et al., 2011).

Alguns dos estudos também afirmaram que esta técnica de observação, foi uma boa estratégia encontrada para introduzir os educandos na profissão, possibilitando exercitar a escrita, ampliando os sentidos e a percepção do ser enfermeiro (Freitas, et al., 2006).

É imprescindível que esta ferramenta também seja utilizada de maneira correta pelos educandos, para que de fato o conhecimento seja produzido e ampliado ao seu desenvolvimento. No aproveitamento de sua escrita para posteriormente usá-la como resgate as teorias, estudos e protocolos abordados.

\section{Limitações do estudo}

Tendo em vista a necessidade atual das boas práticas nos cargos de gestão, sugere-se que sejam realizados mais estudos acerca do assunto para aprofundamento da temática possibilitando maiores reflexões. 


\section{Considerações Finais}

É de suma importância para os educandos e para a sociedade, a aproximação e o vínculo entre as instituições de ensino superior e os serviços de saúde. Além disso, o Diário de Campo é citado como ferramenta relevante em que a partir da escrita das experiências vivenciadas, e dos olhares de diferentes ângulos de cada indivíduo envolvido, torna-se um instrumento valioso para a articulação entre teoria e prática e subjetividade. Também podemos afirmar que as atividades práticas e as observações dos enfermeiros gestores são vistos como essenciais para a experiência da gestão.

Sendo assim, podemos concluir que a gestão em saúde sendo abordada com qualidade, através de um vínculo sólido em relação ao ensino-serviço, metodologias com sucesso comprovado e relação teoria-prática na formação dos enfermeiros, os tornarão profissionais qualificados para o bom desempenho em cargos de gerência em qualquer campo de atuação.

O presente estudo visa contribuir com a prática no cenário atual sobre a gestão na formação do enfermeiro, colaborando com a construção desse conhecimento ao reunir evidências científicas criteriosamente selecionadas, tendo em vista uma crescente ocupação de cargos nesta área. Acredita-se também que os resultados do estudo em questão possam contribuir com o auxílio na condução de novas pesquisas sobre a temática, assim como também na realização de novos debates para tomada de decisão sobre o processo formativo dos enfermeiros, norteando sua prática gerencial e proporcionando-lhes maior autonomia.

\section{Referências}

Barreto, R. M. A., Vasconcelos, M. N., Melo, E. S., Araújo, M. A. F., Lira, R. C. M. \& Albuquerque, I. M. A. N. (2018). Dimensões gerenciais na formação acadêmica de enfermagem: uma revisão integrativa. Rev. Eletr. Enf., 20 (20), 1-17.

Brasil. Resolução CNE/CES n 3 3, de 7 novembro de 2001 (2001). Estabelece Diretrizes Curriculares Nacionais do Curso de Graduação em Enfermagem. Diário Oficial da União. Brasília, DF: Conselho Nacional de Educação.

Erdmann, A. L., Andrade, S. R., Santos, J. L. G. \& Oliveira, R. J. T. (2011). Perfil dos egressos de gerenciamento de enfermagem dos Programas da área de Enfermagem da Região Sul. Rev. da Esc. de Enferm., 45 (Especial), 1551-1557.

Esteves, L. S. F., Cunha, I. C. K. O., Bohomol, E. \& Negri, E. C. (2018) Supervised internship in undergraduate education in nursing: integrative review. Rev Bras Enferm [Internet]. 71(Suppl 4):1740-50. [Thematic issue: Education and teaching in Nursing] DOI: http://dx.doi.org/10.1590/0034-7167-2017-0340

Freitas, M. E. A., Spagnol, C. A. \& Camargos, A. T. (2006). Observação e diário de campo: técnicas utilizadas no estágio da disciplina administração de enfermagem. Rev. Baiana de Enferm., 20 (3), 11-18.

Garcia, I. M., Borges, T. A. P., Pimentel, R. R. S. \& Vannuchi, M. T. O. (2018). Percepção do discente de enfermagem na construção do seu conhecimento no contexto da metodologia ativa. Revista Eletrônica Acervo Saúde, 11(2), e127. https://doi.org/10.25248/reas.e127.2019

Healthcare, B. V. (2013). Critcal Appraisal Skills Programme (CASP). https://casp-uk.net/casp-tools-checklists/

Heck, R.M., Jardim, V.R., Dilélio, A. S. \& Silva, S. J. (2009). Uso de metodologia ativa na disciplina gerenciamento de enfermagem em saúde coletiva da FEO/UFPEL. Rev. Eletr. de Enferm., 11 (2), 429-434.

Magnago, C. \& Pierantoni, C.R. (2020). A formação de enfermeiros e sua aproximação com os pressupostos das Diretrizes Curriculares Nacionais (DCN) e da Atenção Básica. Ciência e Saúde Coletiva, 25 (1), 15-24.

Mendes, K. D. S.; Silveira, R. C. C. P., \& Galvão, C. M. (2008). Revisão integrativa: método de pesquisa para a incorporação de evidências na saúde e na enfermagem. Texto Contexto Enferm, 17 (4), 758-764.

Messias, I. M. de O., de Souza Leal, J. O., Gomes Ferreira, A., da Silva Filho, J. F., \& Brando Messias, J. (2021). Enade à luz das Diretrizes Curriculares Nacionais do Curso de Graduação em Enfermagem. South American Journal of Basic Education, Technical and Technological, 8(2), 295-313. Recuperado de https://periodicos.ufac.br/index.php/SAJEBTT/article/view/4091

Rieg, D. L., Scramim, F. C. L., Raimundo, D. O., Zau, V. Z. \& Calazans, W. R. (2014). Aplicação de procedimentos do planejamento estratégico situacional (PES) para estruturação de problemas no âmbito empresarial: estudos de casos múltiplos. Gest. Prod., 21 (2), $417-431$.

Rigobello, J. L., Bernardes, A., Moura, A. A., Zanetti, A. C. B., Gabriel, C. S. \& Laus, A. M. (2018). Ações assistenciais e gerenciais desenvolvidas no Estágio Curricular Supervisionado: impressão dos atores envolvidos. Rev. da Esc. de Enferm., 52 e03369,1-9.

Santos, C. M. C., Pimenta, C. A. M. \& Nobre, M. R. C. (2007). A estratégia pico para a construção da pergunta de pesquisa e busca de evidências. Rev Latinoam Enferm, 15 (3), 1-4.

Santos, J. L. G. et al. (2018). Estratégias Didáticas no Processo de Ensino-Aprendizagem de Gestão em Enfermagem. Texto \& Contexto - Enfermagem [online]. v. 27, n. 2 [Acessado 16 Dezembro 2021] , e1980016. Disponível em: <https://doi.org/10.1590/0104-070720180001980016>. Epub 03 Maio 2018. ISSN 1980265X. https://doi.org/10.1590/0104-070720180001980016. 
Research, Society and Development, v. 10, n. 17, e247101724859, 2021

(CC BY 4.0) | ISSN 2525-3409 | DOI: http://dx.doi.org/10.33448/rsd-v10i17.24859

Silva, V., Mendes, V., Lima, S., Gonçalves, T. P. G., \& Stipp, M. (2021). Educação Permanente na Prática da Enfermagem: Integração entre ensino e serviço. Cogitare Enfermagem, 26. doi:http://dx.doi.org/10.5380/ce.v26i0.71890

Soares, N. A., Silveira, A. P. O., Silveira, B. V., Vieira, J. S., Souza, L. C. B. A., et al. (2011). O diário de campo utilizado como estratégia de ensino e instrumento de análise do trabalho da enfermagem. Rev. Eletr. de Enferm., 13 (4), 665-670.

Sousa, M. T., Silva, M. D. \& Carvalho, R. (2010). Revisão Integrativa: O que é e como fazer. Einstein. 8(1 Pt 1):102-6

Tenório, H. A. A., Souza, I. B., Gomes Junior, E. L., Santos, R. F. E. P., Correia, D. S. et al. (2019). Gestão e gerenciamento de Enfermagem: perspectivas de atuação do discente. Rev Enferm UFPE, 13 e240535.

Ximenes Neto, F. R. G., Lopes Neto, D., Cunha, I. C. K. O., Ribeiro, M. A., Freire, N. P. et al. (2020). Reflexões sobre a formação em Enfermagem no Brasil a partir da regulamentação do Sistema Único de Saúde. Ciênc. e Saúde Coletiva., 25 (1), 37-43. 